\title{
VIABILIDADE ECONÔMICA DO USO DO SISTEMA DE IRRIGAÇÃO POR GOTEJAMENTO NA CULTURA DA CEBOLA ${ }^{1}$
}

\author{
Economic viability of the use of drip irrigation system on onion crop ${ }^{1}$ \\ Renato Carvalho Vilas Boas², Geraldo Magela Pereira ${ }^{3}$, \\ Ricardo Pereira Reis ${ }^{4}$, Joaquim Alves de Lima Junior ${ }^{5}$, Rodrigo Consoni ${ }^{3}$
}

\begin{abstract}
RESUMO
Objetivou-se, com este estudo, avaliar a viabilidade econômica do uso do sistema de irrigação por gotejamento na cultura da cebola. O experimento foi conduzido em canteiros construídos a céu aberto, na área experimental do Departamento de Agricultura/ UFLA, no período de junho a outubro de 2008. O delineamento experimental utilizado foi em blocos casualizados, em esquema fatorial 2 × 6, com quatro repetições. Os tratamentos constituíram-se de duas cultivares de cebola, cultivar híbrida Optima F1 e cultivar não híbrida Alfa Tropical, e seis tensões da água no solo, 15, 25, 35, 45, 60 e $75 \mathrm{kPa}$. A análise econômica da lavoura irrigada foi fundamentada na teoria dos custos de produção e, considerando-se a metodologia aplicada, conclui-se que as despesas com os recursos variáveis foram as que mais oneraram o custo final da cebola em todos os tratamentos estudados. Os custos totais médios foram, inversamente, proporcionais às produtividades dos tratamentos de tensão da água no solo, para ambas as cultivares, indicando uma resposta à escala de produção. Na condição do experimento, a recomendação é de que se adote, como momento de irrigar, a tensão da água no solo de $15 \mathrm{kPa}$ e, como cultivar o híbrido Optima F1, para que se obtenha maior rentabilidade na atividade produtiva. A irrigação por gotejamento na cultura da cebola, adotando-se as tensões da água no solo e as cultivares estudadas neste trabalho, é uma técnica economicamente viável.
\end{abstract}

Termos para indexação: Irrigação localizada, considerações econômicas, Allium cepa L.

\begin{abstract}
The objective of this study was to evaluate the economic feasibility of the use of drip irrigation system on onion crop. The experiment was set at open field beds in the experimental area of the Agricultural Department/UFLA, from June to October 2008. The experimental design was randomized blocks in factorial scheme $2 \times 6$, with four replicates. Treatments consisted of two onion cultivars, the hybrid Optima F1 and the non hybrid Alfa Tropical and six soil water tension values, 15, 25, 35, 45, 60 and $75 \mathrm{kPa}$. The economic analysis of the irrigated farming was based on the production costs theory and considering the used methodology it was concluded that, for all treatment, variable costs were the ones that most affected the final cost of onion harvest. The average total costs were inversely proportional to the yield. In all soil water tension treatments for both cultivars, indicating a scale effect on the economical result. At the conditions of this experiment, the recommendation is to use $15 \mathrm{kPa}$ tension as an indicator of the moment for irrigation, and the hybrid cultivar Optima F1 for higher profitability. The drip irrigation on onion crop, using the soil water tension values tested and both cultivars is a viable economical technique.
\end{abstract}

Index terms: Trickle irrigation, economical aspects, Allium cepa $\mathrm{L}$.

\section{(Recebido em 27 de abril de 2010 e aprovado em 4 de fevereiro de 2011)}

\section{INTRODUÇÃO}

A cebola é considerada a terceira hortaliça mais importante em termos de valor econômico no Brasil (SOUZA; RESENDE, 2002). Em 2008, a produtividade média nacional, de acordo com o Instituto Brasileiro de Geografia e Estatística - IBGE (2009), situou-se em torno de $20.368 \mathrm{~kg}$ $\mathrm{ha}^{-1}$, o que corresponde a 1.018 sacas de $20 \mathrm{~kg}$ por hectare.

Tendo em vista a sua sensibilidade ao déficit hídrico, muitos trabalhos sobre a irrigação da cultura da cebola mostram que a produtividade de bulbos é altamente dependente da quantidade de água aplicada (SANTA OLALLA et al., 1994; ABU AWWAD, 1996; SAHA et al., 1997; KORIEM et al., 1999; SHOCK et al., 2000). Entretanto, em poucos estudos são analisados critérios de manejo da irrigação por gotejamento nessa cultura (CHOPADE et al., 1998; SHOCK et al., 2000; SANTA OLALLA et al., 2004).

Com relação ao Brasil, os sistemas por aspersão ainda são os mais utilizados no cultivo da cebola, destacando-se o convencional, especialmente nas regiões Sul e Sudeste. Nos últimos anos, em grandes áreas, o

${ }^{1}$ Extraído da Tese de Doutorado do primeiro autor apresentada a Universidade Federal de Lavras/UFLA. Trabalho financiado pela FAPEMIG Engenheiro Agrícola, Doutor - Rua João Renato de Pádua, 65 - Vila Murad - 37200-000 - Lavras,MG - renatovilasboas@yahoo.com.br ${ }^{3}$ Universidade Federal de Lavras/UFLA - Departamento de Engenharia/DEG - Lavras, MG

${ }^{4}$ Universidade Federal de Lavras/UFLA - Departamento de Administração e Economia/DAE - Lavras, MG

5Universidade Federal Rural da Amazônia/UFRA - Departamento de Ciências Exatas/DCE - Paragominas, PA 
sistema pivô central vem sendo utilizado com sucesso (COSTA et al., 2002). No entanto, em virtude da preocupação, em nível mundial, com a questão do gerenciamento, conservação e economia dos recursos hídricos, tem sido recomendado, para a grande maioria das culturas, o uso do método de irrigação localizada (sistemas de microaspersão e gotejamento), por ser mais eficiente na aplicação de água e de fertilizantes (NOGUEIRA et al., 1998).

O sistema de irrigação por gotejamento apresenta muitas vantagens, dentre elas podem-se destacar: maior eficiência no uso da água, maior produtividade, maior eficiência na adubação (fertilizantes podem ser aplicados via água de irrigação) e no controle fitossanitário, economia de mão-de-obra, redução dos gastos com energia e possibilidade de automação.

Apesar das inúmeras vantagens que o sistema oferece, o gotejamento não tem sido utilizado para irrigação da cultura da cebola no Brasil, com exceção de algumas pequenas áreas de observação. No entanto, nos Estados Unidos, já existem grandes áreas, em escala comercial, cultivadas com a cebola irrigadas pelo sistema de gotejamento. Uma das principais limitações é seu alto custo de implantação.

Por ser um sistema fixo, a irrigação por gotejamento exige alto investimento em obras e aquisição de equipamentos para captação, condução, controle e distribuição da água, devendo ser considerados gastos com energia e mão-de-obra para operação e manejo do sistema, que representam importantes custos adicionais à produção. Dessa forma, a determinação da viabilidade econômica de um empreendimento que se inicia, é fundamental para o seu sucesso.

Segundo Silva et al. (2007), a irrigação é uma tecnologia que requer investimentos significativos e está associada à utilização intensiva de insumos agrícolas, tornando importante o estudo econômico dos componentes envolvidos no sistema.

Embora a irrigação possa trazer muitos benefícios aos agricultores, os riscos da adoção de uma agricultura irrigada devem ser criteriosamente estudados e analisados, objetivando-se sempre que o incremento nos rendimentos sejam maiores que os custos de produção. Segundo Silva et al. (2003), o custo da irrigação pode ser previsto por meio de uma avaliação econômica, na qual se estimam todos os dispêndios e retornos anuais esperados no projeto agrícola. O resultado dessa avaliação econômica indicará se é interessante ou não a implantação de um sistema de irrigação.

No Brasil, há uma carência de informações sobre a produção de cebola irrigada por gotejamento, tanto no aspecto do manejo adequado da irrigação, quanto em relação ao estudo de viabilidade econômica dessa tecnologia de produção. Nesse sentido, objetivou-se, com este estudo, avaliar a viabilidade econômica do uso do sistema de irrigação por gotejamento na cultura da cebola.

\section{MATERIAL E MÉTODOS}

Para este estudo de viabilidade, utilizaram-se dados experimentais obtidos entre os meses de junho e outubro de 2008, na área experimental do Departamento de Agricultura da Universidade Federal de Lavras (UFLA), Setor de Olericultura, no município de Lavras, sul de Minas Gerais, tendo como referência as seguintes coordenadas geográficas: latitude $21^{\circ} 14^{\prime} \mathrm{S}$, longitude $45^{\circ} 00^{\prime} \mathrm{W}$ Gr. e 918,8 m de altitude.

O solo foi classificado como Latossolo Vermelho Distroférrico, textura muito argilosa, sendo a curva de retenção da água no solo representada pela Equação 1:

$\theta=0,231+\frac{0,381}{\left[1+(0,485 \cdot|\Psi|)^{1,734}\right]^{0,423}}$

em que: $\theta$ - umidade do solo $\left(\mathrm{cm}^{3} \mathrm{~cm}^{-3}\right)$ e $\Psi$ - tensão da água no solo $(\mathrm{kPa})$.

Foi empregado o delineamento em blocos casualizados (DBC), em esquema fatorial $2 \times 6$, sendo utilizados 12 tratamentos e quatro repetições. Os tratamentos constituíram-se de duas cultivares de cebola de ciclo precoce, cultivar não híbrida Alfa Tropical (A) e cultivar híbrida Optima $\mathrm{F} 1(\mathrm{O})$ e seis tensões da água no solo, $15,25,35,45,60$ e $75 \mathrm{kPa}$ como indicativo do momento de irrigar (tensão crítica). Os tratamentos foram, assim, representados: A15, A25, A35, A45, A60, A75, O15, O25, O35, O45, O60 e 075.

Para monitorar o estado de energia da água no solo, foi instalado um conjunto com três tensiômetros por parcela (dois a $0,15 \mathrm{~m}$ de profundidade para monitorar a irrigação e um a $0,30 \mathrm{~m}$ de profundidade para verificar a ocorrência de percolação), sendo que, para cada tratamento, os conjuntos de tensiômetros foram instalados em duas das quatro repetições. As leituras nos tensiômetros foram realizadas, utilizando-se um tensímetro digital de punção.

As parcelas experimentais tiveram dimensões de $1,20 \mathrm{~m}$ de largura por $1,40 \mathrm{~m}$ de comprimento. Foram utilizadas quatro linhas de plantas, espaçadas de $0,20 \mathrm{~m}$ entre si e 0,10 m entre plantas. Foram consideradas úteis as plantas das linhas centrais e descartadas, nessas linhas, 
duas plantas no início e duas no final (parcela útil com 20 plantas).

Após o transplante das mudas, feito aos 40 dias após a semeadura, a irrigação foi realizada por microaspersão com o tape SANTENO ${ }^{\circledR}$. Esse sistema foi usado até 14 dias após o transplante, período esse necessário para o pegamento e a climatização das mudas, no campo. Na diferenciação dos tratamentos, utilizou-se um sistema de irrigação por gotejamento, sendo os emissores autocompensantes do tipo in-line, modelo NAAN PC com vazão nominal de $1,6 \mathrm{~L} \mathrm{~h}^{-1}$ e distanciados entre si a $0,30 \mathrm{~m}$. O tubogotejador ficou posicionado na parcela, de forma a atender duas fileiras de plantas, trabalhando com pressão de serviço de $140 \mathrm{kPa}$. Na avaliação do sistema, obteve-se um coeficiente de uniformidade de distribuição de água (CUD) de 94,3\%. Suspendeu-se a irrigação da cultura sete dias antes de cada colheita.

Buscava-se, em todas as irrigações, elevar à capacidade de campo a umidade correspondente à tensão verificada no momento de irrigar. O momento de irrigar foi estabelecido como aquele em que, pelo menos três dos tensiômetros de decisão (instalados a $0,15 \mathrm{~m}$ de profundidade), atingiam a tensão crítica estabelecida para cada tratamento. Calculou-se o tempo de funcionamento do sistema de irrigação a partir da lâmina bruta, de acordo com Cabello (1996), considerando-se a profundidade efetiva do sistema radicular igual a $0,30 \mathrm{~m}$. A eficiência de aplicação de água do sistema de irrigação foi adotada como $90 \%$.

Utilizou-se, para a estimativa dos custos de produção, o procedimento econômico, em que se considera o cálculo da depreciação e do custo alternativo (REIS, 2007).

Para estimar o custo de produção, neste trabalho, foram utilizados valores aproximados em reais $(\mathrm{R} \$)$, com base nas seguintes informações: área cultivada com cebola de 1,0 ha, período de uma safra e nos custos fixos e variáveis.

O custo necessário, para substituir os bens de capital, quando tornados inúteis, seja pelo desgaste físico ou econômico, foi definido como a depreciação. O método utilizado foi o linear, considerando-se o prazo de 110 dias (0,30 anos), referente ao ciclo médio de cultivo, no campo, das duas cultivares de cebola utilizadas neste experimento, podendo ser mensurado pela Equação 2:

$\mathrm{D}=\left(\frac{\mathrm{Va}-\mathrm{Vr}}{\mathrm{Vu}}\right) \cdot \mathrm{P}$

em que: $\mathrm{D}$ - depreciação ( $\mathrm{R} \$$ ); Va - valor atual do recurso (R\$); Vr- valor residual (valor de revenda ou valor final do bem após utilizado racionalmente) $(\mathrm{R} \$)$; $\mathrm{Vu}$ - vida útil (período em que determinado bem é utilizado na atividade) (anos) e $\mathrm{P}$ - período de análise (anos).

Considerou-se, para efeito da análise do custo alternativo dos recursos fixos de produção alocados no cultivo da cebola, a taxa de juros real de $6 \%$ a.a. Em seu cálculo, utilizou-se a Equação 3:

$$
\mathrm{CA}_{\text {fixo }}=\left(\frac{\mathrm{Vu}-\mathrm{I}}{\mathrm{Vu}}\right) \cdot \mathrm{Va} \cdot \mathrm{Tj} \cdot \mathrm{P} \quad \mathrm{CA}_{\text {fixo }}
$$

em que: $\mathrm{CA}_{\text {fixo }}$ - custo alternativo fixo (R\$); I - idade média de uso do bem (anos) e $\mathrm{Tj}$ - taxa de juros (decimal).

Para simplificar o cálculo do $\mathrm{CA}_{\text {fixo }}$, considerou-se a idade média de uso dos recursos fixos como $50 \%$ da vida útil $(\mathrm{Vu})$, que resulta na metade do valor atual do recurso (Va), multiplicado pela taxa de juros (Tj) e pelo período de análise (P), conforme a Equação 4:

$$
\mathrm{CA}_{\text {fixo }}=\frac{\mathrm{Va}}{2} \cdot \mathrm{Tj} \cdot \mathrm{P}
$$

Considerou-se, para o cálculo do custo alternativo dos recursos variáveis aplicados na cultura estudada, a taxa de juros real de 6\% a.a. e utilizada a Equação 5:

$$
\mathrm{CA}_{\text {var }}=\frac{\mathrm{V}_{\text {gasto }}}{2} \cdot \mathrm{Tj}
$$

em que: $\mathrm{CA}_{\mathrm{VAR}}$ - custo alternativo variável $(\mathrm{R} \$)$ e $\mathrm{V}_{\text {gasto }}$ desembolso financeiro realizado pelo produtor, para adquirir insumos e serviços necessários para a produção agrícola $(\mathrm{R} \$)$.

Para o cálculo de cada recurso fixo, foram somados à depreciação, o custo alternativo do fator produtivo. Os itens considerados nos custos fixos e o procedimento de operacionalização foram:

a) Terra: não se deprecia, uma vez que, parte da hipótese de que o agricultor adota um manejo de solo adequado. $\mathrm{O}$ valor considerado foi o custo alternativo baseado no aluguel da terra explorada. $\mathrm{O}$ valor do aluguel foi de $\mathrm{R} \$ 80,00$ para um hectare e por um mês, conforme citado nos índices de preços agrícolas do Departamento de Administração e Economia da Universidade Federal de Lavras (DAE/UFLA).

b) Bandejas de poliestireno expandido com 200 células: o gasto com as bandejas foi de $\mathrm{R} \$ 21.600,00$, referente à aquisição de 2.400 unidades para a produção 
de 480.000 mudas (semeadura de $20 \%$ a mais, visando a garantir as mudas necessárias ao transplante). Realizouse a depreciação das bandejas em 0,30 anos, considerandose como vida útil o período de 5 anos.

c) Calagem: o gasto com calagem neste trabalho foi de $\mathrm{R} \$ 237,50$ ha $^{-1}$ a cada 2 anos.

d) Imposto Territorial Rural (ITR): esse recurso não é alterado em curto prazo, pois, seu valor é constante no ano. $\mathrm{O}$ valor considerado foi de $\mathrm{R} \$ 0,11$ para um hectare $\mathrm{e}$ por um ano, conforme citado nos índices de preços agrícolas do DAE/UFLA.

e) Sistema de irrigação: o valor de um sistema de irrigação é altamente influenciado pelas condições do local e pelos equipamentos utilizados. Neste trabalho, considerou-se um projeto com as seguintes características: conjunto motobomba de $5 \mathrm{cv}$, chave de partida direta com contator e relé, Controlador Lógico Programável, injetor de fertilizantes tipo venturi com bomba reforço, válvulas de ar e vácuo, válvulas de comando elétrico (solenoides), válvula de alívio, adutora de aço zincado de $100 \mathrm{~m}$ até o cabeçal de controle, tubulação de PVC do cabeçal até os setores, tubogotejador autocompensante modelo NAAN PC com vazão nominal de 1,6 L h ${ }^{-1}$ e DN 16 mm (distanciados entre si a $0,30 \mathrm{~m}$ ), 2 filtros de disco com retrolavagem automática e desnível do terreno de $40 \mathrm{~m}$. A vida útil considerada foi de 15 anos.

f) Custo alternativo: o cálculo do custo alternativo, para cada um dos recursos do custo fixo, foi feito considerando a taxa de juros real de $6 \%$ a.a.

O desembolso realizado para a aquisição de produtos e serviços, somado ao custo alternativo, serviu como base de cálculo para o custo de cada recurso variável. Os recursos variáveis e a forma de operacionalização utilizada foram:

a) Insumos: corresponde ao gasto com a aquisição de sementes, substrato, fertilizantes químicos, defensivos e espalhante adesivo. $\mathrm{O}$ valor unitário considerado foi aquele citado nos índices de preços agrícolas do DAE/ UFLA, e a quantidade usada de cada insumo foi baseada nas quantidades utilizadas no experimento e, conforme descrito, em Vidigal et al. (2001).

b) Mão-de-obra: os custos com mão-de-obra referem-se à operação do sistema de irrigação, implantação da cultura (formação de mudas, preparo de canteiros, adubação de plantio e transplante de mudas), condução (adubações de cobertura e aplicação de defensivos), colheita (arranquio e enleiramento), limpeza (toalete), ensacamento e transporte dentro da propriedade. $\mathrm{O}$ valor unitário considerado foi aquele citado nos índices de preços agrícolas do DAE/UFLA, e as quantidades usadas de cada serviço foram adotadas segundo Vidigal et al. (2001) e Agrianual (2009).

c) Máquinas e implementos: foram computados os gastos com aluguel de máquinas e implementos utilizados na preparação do terreno (aração e gradagem) e no transporte dentro da propriedade. O valor unitário considerado foi aquele citado nos índices de preços agrícolas do DAE/UFLA, e as quantidades utilizadas de cada recurso foram adotadas segundo Vidigal et al. (2001) e Agrianual (2009).

d) Despesas com administração: foram considerados os gastos com mão-de-obra administrativa, assistência técnica e impostos (2,3\% da receita total), cujos valores unitários e quantidades usadas foram adotados, conforme descrito em Agrianual (2009).

e) Despesas gerais: referem-se aos gastos com sacarias de $20 \mathrm{~kg}$ para o acondicionamento e transporte da cebola, e as quantidades utilizadas em função das produtividades médias observadas em cada tratamento do experimento.

f) Energia: o custo com energia foi calculado, conforme a Equação 6, sugerida por Mendonça (2001).

$\mathrm{CE}=\mathrm{V}_{\mathrm{kWh}} \cdot \mathrm{T} \cdot \frac{736 \cdot \mathrm{Pot}}{1000 \cdot \eta}$

em que: $\mathrm{CE}$ - custo com energia ( $\mathrm{R} \$) ; \mathrm{V}_{\mathrm{KWh}}$ - valor do $\mathrm{kWh}(\mathrm{R} \$) ; \mathrm{T}$ - tempo total de funcionamento do sistema de irrigação (h), variável para cada tratamento; Pot potência do conjunto motobomba (cv) e $\eta$ - rendimento do conjunto motobomba (decimal).

g) Custo alternativo: para o cálculo do custo alternativo, a cada item dos recursos variáveis, foi considerada a taxa de juros real de $6 \%$ a.a.

$\mathrm{O}$ custo econômico foi obtido pela soma entre o custo operacional e o custo alternativo. O custo operacional foi dividido em custo operacional fixo (CopF), composto pelas depreciações e custo operacional variável (CopV), constituído pelos desembolsos. O custo operacional total (CopT) constituiu-se da soma do custo operacional fixo e operacional variável.

Ao se fazer a análise econômica da atividade produtiva, podem-se encontrar diversas condições, dependendo da posição do preço (ou receita média), em relação aos custos e cada qual sugerindo uma particular interpretação. Para a realização dessa análise, foram consideradas as situações de análise econômica e operacional da atividade produtiva, descritas por Reis (2007). 
No presente trabalho, o critério adotado para correção de valores foi o de preço único. Dessa forma, foram somadas as quantidades de recursos utilizados durante o ciclo da cultura da cebola e o resultado foi multiplicado pelo preço vigente em determinada data que, nesse caso, foi em novembro de 2009. Sendo assim, o preço do produto adotado para a análise foi de $\mathrm{R} \$ 26,20$ por saca de $20 \mathrm{~kg}$, conforme sugerido pelo Centro de Estudos Avançados em Economia Aplicada da Escola Superior de Agricultura "Luiz de Queiroz"/Universidade de São Paulo.

\section{RESULTADOS E DISCUSSÃO}

A lâmina total de água, bem como a produtividade média de bulbos comerciais obtida pelas duas cultivares de cebola, em função das diferentes tensões da água no solo, são apresentadas na Tabela 1. Torna-se importante salientar que, a lâmina total de água corresponde à soma entre as lâminas de irrigação aplicadas durante a diferenciação dos tratamentos, a lâmina de $41,4 \mathrm{~mm}$, aplicada durante o período de pegamento das mudas, e a precipitação total de $109,3 \mathrm{~mm}$, ocorrida durante o experimento.

De acordo com os resultados obtidos, observa-se um aumento significativo na produtividade, em sc ha-1 ${ }^{-1}$ de bulbos de cebola, em função do decréscimo das tensões da água no solo estudadas, sendo que, para as lâminas de 615,2 e 603,6 mm, referentes à tensão de $15 \mathrm{kPa}$, a produtividade média de bulbos comerciais chegou a 1.974 sc ha-1 para a cultivar Alfa Tropical e $2.891 \mathrm{sc} \mathrm{ha}^{-1}$ para o híbrido Optima F1, respectivamente.
Analisados critérios de manejo da irrigação por gotejamento na cultura da cebola, Chopade et al. (1998), Shock et al. (2000) e Santa Olalla et al. (2004), também, obtiveram melhores produtividades de bulbos, quando o solo foi mantido, constantemente, com alto teor de água.

Na Tabela 2, são apresentados os percentuais de participação dos itens que compõem os custos totais de produção de cebola para todos os tratamentos estudados.

Nota-se, para ambas as cultivares estudadas, que os tratamentos de irrigação apresentaram uma diminuição da participação percentual dos custos fixos e um aumento da participação percentual dos custos variáveis, em função da diminuição da tensão da água no solo e, consequentemente, do aumento das lâminas de água aplicadas, as quais apresentaram valores crescentes de produtividades médias de bulbos comerciais (Tabela 1).

Em todos os tratamentos estudados, o item que teve maior participação no custo fixo foi bandejas (200 células), enquanto que os gastos com os itens insumos e mão-de-obra foram os que apresentaram maior participação na formação do custo variável.

No tratamento $\mathrm{O} 15$, a participação do item bandejas (200 células), no custo total de produção, foi de $6,74 \%$, destacando-se com o menor percentual desse recurso nos custos fixos, quando comparado aos demais tratamentos. Já, no tratamento A75, esse item apresentou o maior percentual desse recurso nos custos fixos $(9,06 \%$ do custo total).

O valor pago pela energia elétrica representou $7,13 \%$ do custo total médio de cada saca de cebola produzida

Tabela 1 - Lâmina total de água e produtividade média de bulbos comerciais de duas cultivares de cebola, em função das diferentes tensões da água no solo.

\begin{tabular}{ccc}
\hline Tratamentos experimentais & Lâmina total de água $(\mathrm{mm})$ & Produtividade média de bulbos comerciais $\left(\mathrm{sc} \mathrm{ha}^{-1}\right)$ \\
\hline A15 & 615,2 & 1.974 \\
A25 & 574,1 & 1.816 \\
A35 & 543,4 & 1.804 \\
A45 & 506,2 & 1.759 \\
A60 & 390,9 & 1.463 \\
A75 & 276,9 & 1.228 \\
O15 & 603,6 & 2.891 \\
O25 & 514,1 & 2.516 \\
O35 & 447,2 & 2.172 \\
O45 & 398,9 & 1.988 \\
O60 & 360,1 & 1.902 \\
O75 & 245,4 & 1.366 \\
\hline
\end{tabular}


para o tratamento A15. Esse tratamento foi o que recebeu a maior quantidade de água e, por consequência, maior foi o número de horas de funcionamento do sistema de irrigação.

Entre os tratamentos aplicados, o O15 apresentou a maior participação dos custos variáveis no custo total de produção, destacando-se: despesas com administração $(10,87 \%)$ e despesas gerais $(8,28 \%)$. Esse tratamento foi o que apresentou a maior produtividade de bulbos comerciais de cebola e, por consequência, maiores foram os custos com colheita, administração e impostos.

Os resultados dos custos médios de produção de cebola, em relação aos diferentes tratamentos experimentais, são apresentados na Tabela 3. De acordo com os valores desta tabela, pode-se notar que os custos totais médios, tanto econômicos quanto operacionais, apresentaram um aumento de valor, à medida que se aumentou a tensão da água no solo, para ambas as cultivares. A diferença entre o custo econômico e o custo operacional é o custo alternativo do sistema produtivo, adotado nesse estudo.
Observa-se, também, que os custos fixos e variáveis médios diminuíram à medida que a produtividade aumentava, sendo os menores valores apresentados pelo tratamento $\mathrm{O} 15$, cujos custos fixos e variáveis médios foram de $\mathrm{R} \$ 0,72 \mathrm{sc}^{-1}$ e $\mathrm{R} \$ 6,53 \mathrm{sc}^{-1}$, respectivamente, conforme apresentado na Tabela 3 .

Para a realização do estudo econômico simplificado, foram utilizados os dados contidos na Tabela 3, considerando como preço médio da saca de $20 \mathrm{~kg}$ de cebola o valor de $\mathrm{R} \$ 26,20$, correspondente ao período de novembro de 2009.

No estudo econômico efetuado, observa-se que todos os tratamentos experimentais apresentaram receita média (RMe) superior aos custos totais médios (CTMe), indicando haver situações de lucro econômico ( $\mathrm{RMe}>$ $\mathrm{CTMe}$ ). Essa é uma situação em que o investimento paga todos os recursos aplicados na atividade econômica e proporciona um lucro adicional, superior ao de outras alternativas de mercado. A tendência em médio e longo prazo é de expansão e entrada de novas empresas para a atividade, atraindo investimentos competitivos.

Tabela 2 - Percentagem dos custos fixos e variáveis da produção de duas cultivares de cebola, em diferentes tratamentos de tensão da água no solo.

\begin{tabular}{ccccccccccccc}
\hline $\begin{array}{c}\text { Custos Fixos e Variáveis } \\
\text { Totais }{ }^{1}\end{array}$ & \multicolumn{10}{c}{ \% do Custo Total } \\
\cline { 2 - 13 } & A15 & A25 & A35 & A45 & A60 & A75 & O15 & O25 & O35 & O45 & O60 & O75 \\
\hline Terra & 2,30 & 2,34 & 2,35 & 2,37 & 2,47 & 2,56 & 1,91 & 1,98 & 2,04 & 2,07 & 2,10 & 2,21 \\
Bandejas (200 células) & 8,11 & 8,26 & 8,30 & 8,38 & 8,73 & 9,06 & 6,74 & 6,99 & 7,20 & 7,33 & 7,41 & 7,80 \\
Calagem & 0,21 & 0,21 & 0,21 & 0,21 & 0,22 & 0,23 & 0,17 & 0,18 & 0,18 & 0,19 & 0,19 & 0,20 \\
ITR & 0,00 & 0,00 & 0,00 & 0,00 & 0,00 & 0,00 & 0,00 & 0,00 & 0,00 & 0,00 & 0,00 & 0,00 \\
Sistema de irrigação & 1,35 & 1,37 & 1,38 & 1,39 & 1,45 & 1,50 & 1,12 & 1,16 & 1,19 & 1,21 & 1,23 & 1,29 \\
\hline CFT & 11,97 & 12,18 & 12,24 & 12,35 & 12,87 & 13,36 & 9,94 & 10,30 & 10,61 & 10,81 & 10,92 & 11,50 \\
\hline Insumos & 28,99 & 29,49 & 29,64 & 29,91 & 31,16 & 32,35 & 35,58 & 36,86 & 37,98 & 38,67 & 39,09 & 41,17 \\
Mão-de-obra & 29,36 & 29,88 & 30,03 & 30,30 & 31,57 & 32,77 & 24,40 & 25,27 & 26,04 & 26,52 & 26,80 & 28,23 \\
Máquinas e implementos & 5,06 & 5,15 & 5,18 & 5,22 & 5,44 & 5,65 & 4,21 & 4,36 & 4,49 & 4,57 & 4,62 & 4,87 \\
Despesas com & 9,90 & 9,52 & 9,53 & 9,45 & 8,75 & 8,17 & 10,87 & 10,14 & 9,39 & 8,99 & 8,81 & 7,50 \\
administração & 6,80 & 6,37 & 6,36 & 6,26 & 5,42 & 4,72 & 8,28 & 7,46 & 6,64 & 6,19 & 5,98 & 4,52 \\
Despesas gerais & 7,13 & 6,63 & 6,24 & 5,72 & 4,01 & 2,20 & 5,92 & 4,80 & 4,06 & 3,46 & 2,98 & 1,42 \\
Energia & 0,79 & 0,79 & 0,79 & 0,79 & 0,78 & 0,78 & 0,81 & 0,80 & 0,80 & 0,80 & 0,80 & 0,79 \\
\hline Custo alternativo & 88,03 & 87,82 & 87,76 & 87,65 & 87,13 & 86,64 & 90,06 & 89,70 & 89,39 & 89,19 & 89,08 & 88,50 \\
\hline CVT & 100,0 & 100,0 & 100,0 & 100,0 & 100,0 & 100,0 & 100,0 & 100,0 & 100,0 & 100,0 & 100,0 & 100,0 \\
\hline CT & & &
\end{tabular}

${ }^{1} \mathrm{CFT}$ - custo fixo total (já incluído o custo alternativo); CVT - custo variável total e CT - custo total. 
Tabela 3 - Custos econômicos e operacionais médios ${ }^{1}$ da produção de duas cultivares de cebola, em $\mathrm{R} \$ \mathrm{sc}^{-1} \mathrm{de} 20 \mathrm{~kg}$, em diferentes tratamentos de tensão da água no solo.

\begin{tabular}{ccccccc}
\hline Tratamentos & CFMe & CVMe & CTMe & CopFMe & CopVMe & CopTMe \\
\hline A15 & 1,06 & 7,77 & 8,82 & 0,77 & 7,70 & 8,46 \\
A25 & 1,15 & 8,28 & 9,42 & 0,83 & 8,20 & 9,04 \\
A35 & 1,16 & 8,28 & 9,44 & 0,84 & 8,21 & 9,05 \\
A45 & 1,18 & 8,41 & 9,59 & 0,86 & 8,33 & 9,19 \\
A60 & 1,42 & 9,65 & 11,07 & 1,03 & 9,56 & 10,59 \\
A75 & 1,70 & 11,01 & 12,70 & 1,23 & 10,91 & 12,14 \\
O15 & 0,72 & 6,53 & 7,25 & 0,52 & 6,47 & 6,99 \\
O25 & 0,83 & 7,21 & 8,04 & 0,60 & 7,15 & 7,75 \\
O35 & 0,96 & 8,08 & 9,04 & 0,70 & 8,01 & 8,70 \\
O45 & 1,05 & 8,65 & 9,70 & 0,76 & 8,57 & 9,33 \\
O60 & 1,10 & 8,94 & 10,03 & 0,80 & 8,86 & 9,65 \\
O75 & 1,53 & 11,73 & 13,26 & 1,11 & 11,63 & 12,74 \\
\hline
\end{tabular}

${ }^{1} \mathrm{CFMe}$ - custo fixo médio; CVMe - custo variável médio; $\mathrm{CTMe}$ - custo total médio; CopFMe - custo operacional fixo médio; CopVMe custo operacional variável médio; CopTMe - custo operacional total médio.

\section{CONCLUSÕES}

As despesas com os recursos variáveis foram as que mais oneraram o custo final da produção de cebola em todos os tratamentos estudados.

Os custos totais médios foram, inversamente, proporcionais às produtividades dos tratamentos de tensão da água no solo, para ambas as cultivares, indicando uma resposta à escala de produção.

$\mathrm{Na}$ condição do experimento a recomendação é de que se adote, como momento de irrigar, a tensão da água no solo de $15 \mathrm{kPa}$, e como cultivar, o híbrido Optima F1, para que se obtenha maior rentabilidade na atividade produtiva.

A irrigação por gotejamento na cultura da cebola, adotando-se as tensões da água no solo e as cultivares estudadas neste trabalho, é uma técnica economicamente viável.

\section{AGRADECIMENTOS}

À FAPEMIG - Fundação de Amparo à Pesquisa do Estado de Minas Gerais, pelo financiamento do projeto de pesquisa (Processo: CAG-APQ-1569-3.12/07) e pela concessão das bolsas de Doutorado e Iniciação Científica e ao CNPq - Conselho Nacional de Pesquisa e Desenvolvimento Científico e Tecnológico, pela concessão da bolsa de Produtividade em Pesquisa.

\section{REFERÊNCIAS BIBLIOGRÁFICAS}

ABU AWWAD, A.M. Irrigation water management for onion trickle irrigated with saline drainage water. Dirasat
Series B. Pure and Applied Sciences, Amman, v.23, n.1, p.46-54, 1996.

AGRIANUAL. Anuário da agricultura brasileira. São

Paulo: FNP Consultoria e Comércio, 2009.

CABELLO, F.P. Riegos localizados de alta frecuencia (RLAF) goteo, microaspersión, exudación. 3.ed. Madrid: Mundi, 1996. 511p.

CHOPADE, S.O.; BANSODE, P.N.; HIWASE, S.S. Studies on fertilizer and water management to onion. PKV Research Journal, Akola, v.22, p.44-47, 1998.

COSTA, E.L. et al. Irrigação da cebola. Informe Agropecuário, Belo Horizonte, v.23, n.218, p.57-66, 2002.

INSTITUTO BRASILEIRO DE GEOGRAFIA E ESTATÍSTICA. Anuário estatístico do Brasil. Rio de Janeiro, 2009.

KORIEM, S.O.; EL-KOLIEY, M.M.; EL-SHEEKH, H.M. Effect of drought conditions on yield, quality and some water relationships of onion. Journal of Agricultural Sciences, Assiut, v.30, n.1, p.75-84, 1999. 
MENDONÇA, F.C. et al. Evolução dos custos e avaliação econômica de sistemas de irrigação utilizados na cafeicultura. In: SANTOS, C.M. et al. Irrigação da cafeicultura no cerrado. Uberlândia: UFU, 2001. p.45-78.

NOGUEIRA, L.C.; NOGUEIRA, L.R.Q.; MIRANDA, F.R. Irrigação do coqueiro. In: FERREIRA, J.M.S.;

WARWICK, D.R.N.; SIQUEIRA, L.A. A cultura do

coqueiro no Brasil. 2.ed. rev. e ampl. Brasília: Embrapa/ SPI; Aracaju: Embrapa/CPATC, 1998. p.159-187.

REIS, R.P. Fundamentos de economia aplicada. 2.ed. rev. e ampl. Lavras: UFLA/FAEPE, 2007. 95p.

SAHA, U.K. et al. Yield and water use of onion under different irrigation schedules in Bangladesh. Japanese Journal of Tropical Agriculture, Tokyo, v.41, n.4, p.268274, 1997.

SANTA OLALLA, F.M.; DOMINGUEZ-PADILLA, A.; LOPEZ, R. Production and quality of onion crop (Allium cepa $\mathrm{L}$.) cultivated in semi-arid climate. Agricultural Water Management, Columbus, v.68, p.77-89, 2004.

SANTA OLALLA, F.M.; VALERO, J.A.J.; CORTES, C.F. Growth and production of onion crop (Allium cepa L.) under different irrigation scheduling. European Journal of Agronomy, Córdoba, v.3, n.1, p.85-92, 1994.

SHOCK, C.C.; FEIBERT, E.B.G; SAUNDERS, L.D. Irrigation criteria for drip-irrigated onions. HortScience, Alexandria, v.35, n.1, p.63-66, Feb. 2000.

SILVA, A.L.; FARIA, M.A.; REIS, R.P. Viabilidade técnico-econômica do uso do sistema de irrigação por gotejamento na cultura do cafeeiro. Revista Brasileira de Engenharia Agrícola e Ambiental, Campina Grande, v.7, n.1, p.37-44, 2003.

SILVA, M.L.O. et al. Viabilidade técnica e econômica do cultivo de safrinha do girassol irrigado na região de Lavras, MG. Ciência e Agrotecnologia, Lavras, v.31, n.1, p.200-205, jan./fev. 2007.

SOUZA, R.J.; RESENDE, G.M. Cultura da cebola. Lavras: UFLA, 2002. 115p. (Texto Acadêmico, 21).

VIDIGAL, S.M.; COSTA, E.L.; MENDONÇA, J.L. Cultivo da cebola irrigada na região Norte de Minas Gerais. Belo Horizonte: Epamig, 2001. 36p. (Boletim Técnico, 62). 